\title{
Frontal lobe and long-term memory retrieval analysis during pre-learning stress using EEG signals
}

\author{
Omar Al Shorman', Ahmad Al Shorman² \\ ${ }^{1}$ Electrical Engineering Department, Najran University, Najran, Saudi Arabia \\ ${ }^{2}$ Mechanical Engineering Department, Jordan University of Science and Technology, Irbid, Jordan
}

\begin{tabular}{l}
\hline Article Info \\
\hline Article history: \\
Received Sep 23, 2018 \\
Revised Jan 7, 2019 \\
Accepted Sep 26, 2019 \\
\hline
\end{tabular}

Keywords:

EEG

Frontal lobe

Long-term memory

Pre-Learning stress

\begin{abstract}
This paper uses the EEG analysis to investigate the relationship between pre-learning stress, frontal lobe and long-term memory in the brain. The stress on learning stage is a challenge, especially in academic life. Stress also on learning stage affects the retrieval or recall from the memory. Nowadays; there are many recent works have discovered the relationship between stress, learning and memory performance based on different techniques. Some of these techniques are biological methods. Moreover, these methods have discovered the effect of stress based on hormones levels such as cortisol, or based on physiological effects such as blood pressure. However, these techniques have given conflicting discoveries because of the instability of hormones and an extensive number of related elements. The main aim of this research is to discover the relationship between Pre-learning stress, frontal lobe, and long-term memory retrieval using EEG signals. The experimental results indicate that there is a relationship between theta rhythm in the frontal lobe and long-term memory retrieval during Pre-learning stress.
\end{abstract}

This is an open access article under the CC BY-SA license.

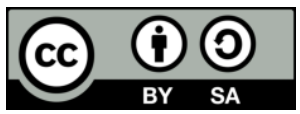

Corresponding Author:

Omar Al Shorman,

Electrical Engineering Department, Najran University, Najran, Saudi Arabia.

Email: omar2007_ahu@yahoo.com

\section{INTRODUCTION}

These days, there are many people in the world suffer from worry and stress through regular daily existence. In 2008, the American Institute of Stress reported that approximately $80 \%$ of college students normally feel stress often in their daily learning ta sks and assignments. Moreover, in 2013, American College Health Association also showed that approximately $30.7 \%$ of undergraduate college students suffer from stress during their academic life. Importantly, these discoveries demonstrate very dangerous risk of stress. Hazardously, chronic stress capable to hurt a hippocampus region in the brain [1,2]. However, the effect of stress for the human body could be physical (i.e. increasing of heart rate), emotional (i.e. anger, anxiety, and depression), behavioral (i.e. lacking of eating and sleeping), and cognitive (i.e. learning and memory problems) [3-5]. Consequently, stress influences the human daily activities such as managing life's tasks, working and educational performance depending on the kind of stressors) [6]. Moreover, when the human faces stress biochemical reactions will be secreted as a response of stressor, which includes increasing of several body hormones' levels such as cortisol.

Stress can also influence short-term memory (STM) and long-term memory (LTM) and for all memory stages: encoding, retention (consolidation), a nd retrieval (recall) [7-11]. Furthermore, there are many factors interferes with studying the affection of stress on learning and memory processes which increase 
the complexity of discovering that affection. On the other hand, the effect of stress on memory (encoding, retention and retrieval) may be varied during the learning or during the time of stressor.

Studying the effect of stress on learning and memory processes from psychobiological point of view have focused on different factors related with stress [12], which includes, nature of stressor and timing of stressor [13], intensity and severity of stressor [14], source of stressor, gender [15], individual differences [16] and learning types [17]. To diagnose the mental disorders and abnormalities for humans; recently, the electroencephalogram (EEG) has become very valuable and vital technique. The main goal of this technique is to measure brain activities. Morover, the desired features could be extracted from the signals, which are recorded from the scalp of the brain.

Nowadays, EEG has been used extensively to detect and study human stress [18]. In EEG analysis, alpha, theta and gamma rhythms have an essential role in human cognitive process and functionality of the brain. Moreover, Theta rhythm is associated and related to memory and learning process, especially for encoding and retrieval phases in all types of memories [19]. In [20], memory retrieval process has been discussed, however, the authors discovered that power of parrietal theta increases for normal memory retrieval. Moreover, In [21], the main power for theta and gamma bands are measured during memory encoding stage. In conclusion, successful encoding is related to decreasing alpha band activity at both pre-frontaland occipital lobes.

Stress assessment based on EEG analysis is discussed in [22], in one hand, busy brain decreases alpha power and increases beta power. In the other hand, the power in left pre-frontal increases during stress $[23,24]$. Until now, studying pre-learning stress using EEG signals still infant and there is a lack of contributes in this regard [25-27]. Twenty-two non-smoking, undergraduate university male healthy students between 18 and 22 of age participated in the experiment. According to exclusion criteria; all subjects must be healthy and none of them has an illness history, acute disorders, chronic disorders, or took medication or drugs. All subjects will sign written informed consent. The experiment conducted afternoon (as known medically the cortisol level is stable in afternoon time). Moreover, cortisol level is high in the morning and low at the night. The experiment consists of two sessions. The participants in the experiment are divided into 2 groups, first group is called control group and second group is called pre-stressed group.

In more details, for the control group; all subjects in this group are learned some neutral content or list of neutral words without facing the stress and in comfortable state. In the pre-stressed group, before the encoding phase in 5 minutes, the subjects were laid under stressor, after that, they are learned some neutral content or list of neutral words. After 2 months (to measure the long term me mory retrieval) with comfortable state, the subjects in both groups a sked verbally to free recall the neutral contents that were learned previously in the encoding phase for 5 minutes. Importantly, 128 channels EEG were used in both groups to record the EEG activities. The main contribution of this paper is to discover the relationship between theta rhythm and frontal lobe during pre-learning stress.

\section{RESEARCH METHOD}

All the latest studies in the effect of stress on memory and learning have investigated the relationships between biological factors (i.e. Hormones) and memory performance. According to these studies, it is observable that there is a conflict in the findings. This research conflict comes from the instability of human hormones. Moreover, there are many factors such as, gender, age, mood, health status, drugs usage, smoking and so forth interferes with studying stress. Importantly, all these factors play a crucial role of inconsistency in the findings and the result. So, the searching for another technique to study stress is becoming very important and useful. However, stress affects learning stage and all memory stages (learning, consolidation and recall) in the brain that depends mainly on the time of the stressor. Moreover, stress may interfere with encoding stage, consolidation stage and retrieval stage as shown in Figure 1.

Retrieval phase of long-term memory is very important. Remembering the information that previously encoded and stored in the memory is very important for the humans. Importantly, enhancement the memory retrieval leads to enhancement human performance and daily activities especially, academic performance for students. Moreover, strong and deep learning (encoding) leads to strong recall (retrieval). That why the motivation of this research comes from. In this research study, EEG analysis is applied to discover the effect of pre-learning stress in EEG bands (alpha, beta, theta and gamma) in the frontal lobe. The methodology of the study is shown in Figure 2. 




Figure 1. The relationship between stress and time of stressor for learning and memory stages

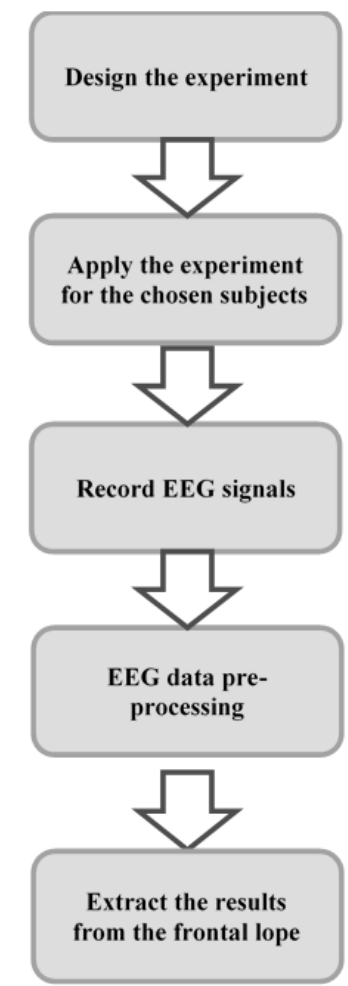

Figure 2. Flow chart for EEG pre-stress a nalysis algorithm

\section{RESULTS AND ANALYSIS}

In order to assess the effect of pre-learning stress; the EEG data [24] has been recorded from the subjects using 128 EEG channels system. The recording process was conducted after two months from the learning session to assess long-term memory. Firstly, notch filter has been used to filter the signal and to remove AC power noise. After that, the filtered signal is passed through band pass filter with cut-off frequency 0.1-30 HZ. As the EEG data is sensitive to other artifacts such as EOG and EMG, Independent component analysis (ICA) is applied to remove these artifacts. EEG Lab MATLAB toolbox for EEG processing is used to process the EEG data. Mean power spectrum for EEG bands theta (4-8 $\mathrm{Hz}$ ) for 128 channels were recorded and extracted. Consequently, the changes in EEG rhythm (theta) in frontal lobe were shown. In addition to the changes in the rhythms, paired sample t-test is used to investigate the significance of the difference in theta band during long-term memory retrieval and eyes open (EO, control) in frontallobe, as shown in Figures 3, 4 and 5. Figure 3 shows the main power for theta band, beta band and alpha band at frontallobe during long-term memory retrieval. 




Figure 3. Mean power of Theta, beta and alpha bands at Frontallobe during LTM retrieval

Figure 4 shows the main power for theta band, beta band and alpha band at frontal lobe during Eyes Open (EO) state. The difference of theta band power between Eyes Open (EO) state and long-term memory retrieval at frontallobe is shown in Figure 5.

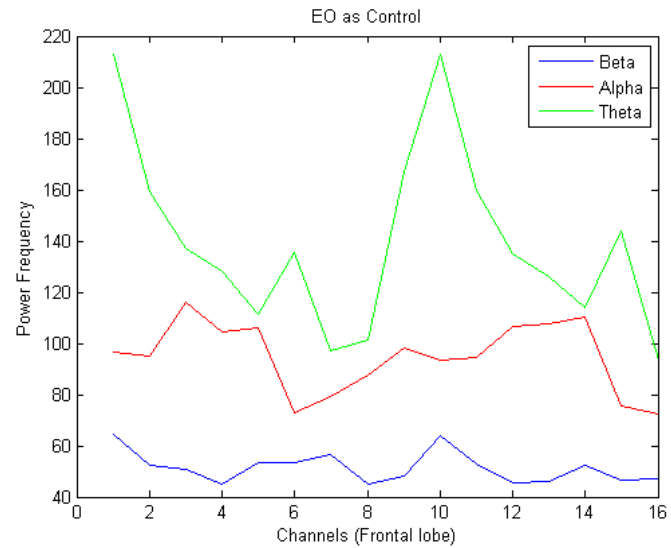

Figure 4. Mean power of Theta, beta and alpha bands at frontal lobe during EO

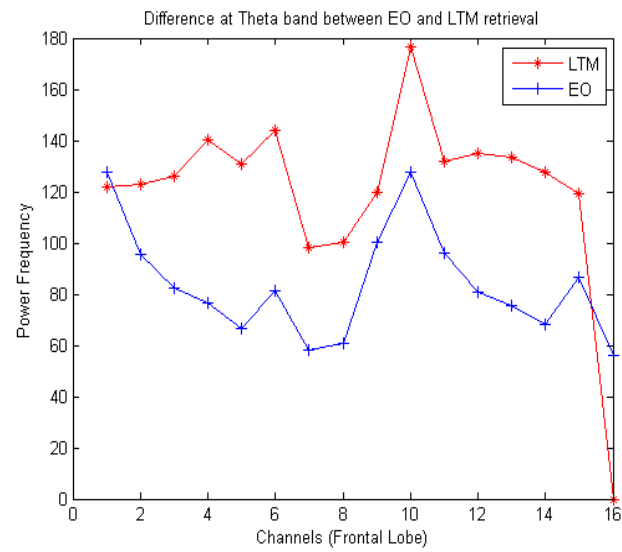

Figure 5. Difference of theta band power between EO and LTM retrieval at frontallobe

\section{CONCLUSION}

In conclusion, the current study shows effects of pre-learning stress in theta rhythm at the frontal lobe in the brain. In general, it has been concluded that the theta band $(4-8 \mathrm{~Hz})$ means power increased at frontal lobe during long term memory retrieval for the pre-stressed group compared with theta power in frontal lobe for the control group. The results suggest that pre-learning stress increases theta main power in the frontal lobe. That also may consider as a long-term memory retrieval performance index. Furthermore, statistically, paired sample t-test showed that the $\mathrm{P}$ value for theta band between long-term memory LTM retrieval for pre-stressed group and EO at frontallobe was equaled to 0.0431 .

\section{REFERENCES}

[1] Lucia P. Pavlova, Dmitrii N. Berlov, Andres Kurismaa., "Dominant and opponent relations in cortical function: An EEG study of exam performance and stress," AIMS Neuroscience, vol. 5, no. 1, pp. 32-55, 2018.

[2] R. N. Goodman, J. C. Rietschel, L. C. Lo, M. E. Costanzo, and B. D. Hatfield, "Stress, emotion regulation and cognitive performance: the predictive contributions of trait and state relative frontal EEG alpha asymmetry," Int J Psychophysiol, vol. 87, no. 2, pp. 115-123, Feb 2013.

[3] P. Bob, "Stress, conflict and the brain," In: Bob, P. (ed.) The Brain and Conscious Unity. Freud's Omega, Springer, New York. pp. 57-68, 2015. 
[4] V. Lai, C. Theppitak, T. Makizuka, Y. Higuchi, M. Movahed, G. Kumudini, et al., "A normal intensity level of psycho-physiological stress can benefit working memory performance at high load," International Journal of Industrial Ergonomics, vol. 44, no .3, pp. 362-367, 2014.

[5] N. Sharma and T. Gedeon, "Objective measures, sensors and computational techniques for stress recognition and classification: a survey," Comput Methods Programs Biomed, vol. 108, no. 3, pp. 1287-1301, Dec 2012.

[6] R. S. Lewis, A. Nikolova, D. J. Chang, and N. Y. Weekes, "Examination stress and components of working memory," Stress, vol. 11, no. 2, pp. 108-114, Mar 2008.

[7] L. Schwabe, M. Joels, B. Roozendaal, O. T. Wolf, and M. S. Oitzl, "Stress effects on memory: an update and integration," Neurosci Biobehav Rev, vol. 36, no. 7, pp. 1740-1749, Aug 2012.

[8] R. N. Goodman, J. C. Rietschel, L. C. Lo, M. E. Costanzo, and B. D. Hatfield, "Stress, emotion regulation and cognitive performance: the predictive contributions of trait and state relative frontal EEG alpha asymmetry," Int J Psychophysiol, vol. 87, no. 2, pp. 115-123, Feb 2013.

[9] B. S. Oken, I. Chamine, and W. Wakeland, "A systems approach to stress, stressors and resilience in humans," Behav Brain Res, vol. 282, pp. 144-154, Apr 2015.

[10] J. S. Olver, M. Pinney, P. Maruff, and T. R. Norman, "Impairments of spatial working memory and attention following acute psychosocial stress," Stress Health, vol. 31, no.2, pp. 115-23, Apr 2015.

[11] W. Skrandies and A. Klein, "Brain activity and learning of mathematical rules--effects on the frequencies of EEG," Brain Res, vol. 1603, pp. 133-140, Apr 72015.

[12] C. E. Cadle and P. R. Zoladz, "Stress time-dependently influences the acquisition and retrieval of unrelated information by producing a memory of its own," Front Psychol, vol. 6, pp. 910, 2015.

[13] S. B. P. a. J. Herbert., "Corticosterone differentially modulates expression of corticotropin releasing factor and arginine vasopressin mRNA in the hypothalamic paraventricular nucleus following either acute or repeated restraint stress," European Journal of Neuroscience, vol. 13, no. 3, 576-584, 2001.

[14] M. Joels, "Corticosteroid effects in the brain: U-shape it," Trends Pharmacol Sci, vol. 27, no. 5, pp. 244-50, May 2006.

[15] V. Luine, "Sex differences in chronic stress effects on memory in rats," Stress, vol. 5, no.3, pp. 205-216, Jul 2009.

[16] J. Davila and R. J. Cobb, "Predicting change in self-reported and interviewer-assessed adult attachment: Tests of the individual difference and life stress models of attachment change," Personality and Social Psychology Bulletin, vol. 29 , no. 7 , pp. 859-870, 2003.

[17] M. Moscovitch, L. Nadel, G. Winocur, A. Gilboa, and R. S. Rosenbaum, "The cognitive neuroscience of remote episodic, semantic and spatial memory," Curr Opin Neurobiol, vol. 16, no. 16, pp. 179-190, Apr 2006.

[18] S. K. Jena, "Examination stress and its effect on EEG," International Journal of Medical Science and Public Health, vol. 4, no. 11, pp.1493-1497, 2015.

[19] L.-T. Hsieh and C. Ranganath, "Frontal midline theta oscillations during working memory maintenance and episodic encoding and retrieval," Neuroimage, vol. 85, no. 2, pp. 721-729, 2014.

[20] J. Jacobs, G. Hwang, T. Curran, and M. J. Kahana, "EEG oscillations and recognition memory: theta correlates of memory retrieval and decision making," Neuroimage, vol. 32, no. 2, pp. 978-987, 2006.

[21] U. Friese, M. Köster, U. Hassler, U. Martens, N. Trujillo-Barreto, and T. Gruber, "Successful memory encoding is associated with increased cross-frequency coupling between frontal theta and posterior gamma oscillations in human scalp-recorded EEG," Neuroimage, vol. 66, pp. 642-647, 2013.

[22] J. F. Alonso, S. Romero, M. R. Ballester, R. M. Antonijoan, and M. A. Mananas, "Stress assessment based on EEG univariate features and functional connectivity measures," Physiol Meas, vol. 36, no. 7, pp. 1351-1365, Jul 2015.

[23] H. Peng, B. Hu, F. Zheng, D. Fan, W. Zhao, X. Chen, et al., "A method of identifying chronic stress by EEG," Personal and Ubiquitous Computing, vol. 17, pp. 1341-1347, 2012.

[24] O AlShorman, T Ali, M Irfan, "EEG Analysis for Pre-learning Stress in the Brain," Asian Simulation Conference, Springer, vol. 752, pp.447-455, 2017.

[25] Fabian Parsia George, Istiaque Mannafee Shaikat, Prommy Sultana Ferdawoos, Mohammad Zavid Parvez, Jia Uddin, "Recognition of emotional states using EEG signals based on time-frequency analysis and SVM classifier," International Journal of Electrical and Computer Engineering (IJECE), vol. 9, no. 2, pp. 1012-1020, 2019.

[26] Sandy Akbar Dewangga, Handayani Tjandrasa, Darlis Herumurti, "Robot motion control using the emotiv EPOC EEG system," Bulletin of Electrical Engineering and Informatics(BEEI) , vol. 7, no. 2, pp. 279-285, 2018.

[27] Mohd Suhaib Kidwai , S. Hasan Saeed, "A novel approach to study the effects of anesthesia on respiratory signals by using the EEG signals," International Journal of Electrical and Computer Engineering (IJECE), vol. 6, no. 2, pp. 117-122, 2017. 